\title{
Deux disques de musique populaire suisse
}

\section{Silvia Delorenzi-Schenkel}

Traducteur : Isabelle Schulte-Tenckhoff

\section{OpenEdition}

\section{Journals}

Édition électronique

URL : http://journals.openedition.org/ethnomusicologie/945

ISSN : 2235-7688

\section{Éditeur}

ADEM - Ateliers d'ethnomusicologie

\section{Édition imprimée}

Date de publication : 1 décembre 1997

Pagination : 366-369

ISBN : 2-8257-0579-9

ISSN : 1662-372X

\section{Référence électronique}

Silvia Delorenzi-Schenkel, «Deux disques de musique populaire suisse », Cahiers d'ethnomusicologie [En ligne], 10 | 1997, mis en ligne le 06 janvier 2012, consulté le 01 mai 2019. URL : http:// journals.openedition.org/ethnomusicologie/945

Ce document a été généré automatiquement le 1 mai 2019.

Tous droits réservés 


\title{
Deux disques de musique populaire suisse
}

\author{
Silvia Delorenzi-Schenkel \\ Traduction : Isabelle Schulte-Tenckhoff
}

\section{RÉFÉRENCE}

Die Volksmusikinstrumente der Schweiz / Les instruments de musique traditionnels suisses / Traditional Swiss Musical Instruments. Enregistrements Télévision suisse romande, 1979-1980. Accompagné d'une brochure trilingue (allemand / français / anglais) illustrée de 52 pages. Recherche et texte (remis à jour) Brigitte Bachmann-Geiser. 1 CD Claves 50-9621, 1996 (rééd.).

Schweizer Volksmusik /Traditional Swiss Music. Tag der Schweizer Volksmusik IMF Luzern. Lieder, Jodel, Rufe, Tänze / Songs, yodels, cow calls, dances. Innerschweiz, Bern, Appenzell, Graubünden, Ticino, Romandie. Enregistrements Radio DRS (Studio Bâle), Lucerne, 1994. Livret bilingue (allemand / anglais) illustré de 27 pages. Sélection musicale et texte Brigitte Bachmann-Geiser. 2 CD Zytglogge ZYT 4532, 1996.

\section{NOTE DE L'ÉDITEUR}

Traduit de l'allemand par Isabelle Schulte-Tenckhoff

1 Ces deux publications sonores ont été réalisées à différents moments et par différentes maisons d'édition. Mais toutes deux portent sur la même matière, à savoir la musique populaire suisse, et ont été conçues par l'ethnomusicologue Brigitte Bachmann-Geiser, qui est aussi l'auteur des notices d'accompagnement. C'est pourquoi j'entreprendrai une comparaison substantielle des deux, plutôt qu'un compte rendu de chaque publication individuelle. Je tiens également à souligner d'entrée que le point de vue adopté ici n'est 
pas seulement celui de l'ethnomusicologue mais aussi celui de l'archiviste et de la documentaliste de supports sonores à la Phonothèque Nationale Suisse.

De janvier 1979 à avril 1980, une équipe de la Télévision suisse romande réalisa une série intitulée Balade à travers la musique populaire suisse, selon une conception élaborée par Brigitte Bachmann-Geiser. Avec la participation de plus de quatre cents musiciens et musiciennes de presque toutes les régions de Suisse, ces films présentent la facture, la technique de jeu et l'utilisation d'une soixantaine d'instruments de musique. Par la suite, la maison Claves publia les matériaux sonores sous forme de deux disques 33 tours, accompagnés d'une brochure en quatre langues richement illustrée. La notice d'accompagnement était basée sur les données recueillies par Brigitte Bachmann-Geiser au cours de recherches menées entre 1971 et 1977 dans toute la Suisse et publiées dans l'ouvrage Die Volksmusikinstrumente der Schweiz (Leipzig/Zürich 1981). En 1993, le diffuseur japonais de Claves publia le matériel pour la première fois sur disque compact. Claves suivit l'exemple en republiant les plages des 33 tours sur $\mathrm{CD}$; à cette occasion il proposa aussi une version mise à jour de la notice d'accompagnement, enrichie de nouvelles illustrations. Cette republication sur un support meilleur et plus durable que le microsillon se justifie sans doute en raison de la pérennité et de la valeur des matériaux sonores. Dans le cas présent, la question du support représente un facteur d'autant plus crucial que tous les exemples sonores sont tirés d'enregistrements professionnels de grande qualité.

De plus, il ne s'agit pas seulement de simples extraits de musique mais de documents sonores soutenus par un travail scientifique. Les instruments sont répartis selon la classification établie par Hornbostel et Sachs, comprenant idiophones, membranophones, cordophones et aérophones. Après une brève présentation de chaque classe, les différents instruments sont décrits du point de vue organologique. Suit l'indication du lieu et de la date de chaque enregistrement, ainsi que de l'identité des interprètes et du titre de la pièce jouée. Pour quelques instruments, on dispose d'une photo qui les illustre en situation de jeu; d'autres font l'objet de dessins. De nombreux instruments - en particulier les idiophones - sont mentionnés par leur appellation locale, par exemple le terme suisse-allemand Chlefeli pour les castagnettes ou l'expression valaisanne Täggi, voire celle de tablèch - terme provenant du dialecte tessinois - pour le tapolet.

Brigitte Bachmann-Geiser n'a pas hésité à inclure dans les idiophones et les aérophones des instruments joués avant tout par les enfants. Dans le cas des idiophones, il s'agit notamment d'instruments bruyants que les enfants font sonner lors de certains rituels coutumiers. Parmi les aérophones simples, joués par les enfants, on trouve soit des rhombes fabriqués en différentes matières naturelles, soit des flûtes et des hautbois en matières végétales.

5 Aux nombreux instruments représentant les quatre classes mais connus également dans d'autres cultures musicales, il faut ajouter ceux qui sont de tradition proprement suisse, comme la pièce de cinq francs et la jatte, avec lesquelles on produit la «ronde de l'écu »qui, en Appenzell, accompagne souvent le yodel naturel (Naturjodel); autre exemple, le cor des Alpes et sa variante coudée, le Büchel. Grâce à ces documents sonores provenant d'enregistrements qui ne remontent pas à plus d'un quart de siècle, il est facile de réfuter l'idée reçue qui veut qu'il n'existe plus guère aujourd'hui de musique traditionnelle en Suisse. Loin d'une collection muséale d'instruments de musique témoignant d'une culture musicale désuète, l'anthologie en question rend hommage à une tradition musicale toujours vivante. C'est là, à mon avis, sa principale contribution, 
laquelle, comme on verra, est largement confirmée par la seconde publication dont il est ici question.

Quelques remarques critiques au sujet de la conception de la notice d'accompagnement s'imposent néanmoins. On l'a dit: pour la publication en $\mathrm{CD}$, le texte de la notice a été remanié. Or, le seul changement pertinent par rapport à la publication initiale est l'adaptation du titre du disque à la terminologie actuellement en vigueur. Au lieu de « Les instruments de musique populaire en Suisse » et « Folk Music Instruments of Switzerland », on lit maintenant «Les instruments de musique traditionnels suisses» et «Traditional Swiss Musical Instruments ». Mais le choix des illustrations de la réédition laisse à désirer. Dans bien des cas, on retrouve celles de la publication antérieure, mais elles ont été reproduites sous un angle différent qui ne permet pas nécessairement de mieux distinguer le sujet. De plus, comme un livret de CD est de format plus petit qu'un livret de disque 33 tours, il fallait réduire les illustrations. En revanche, la modification qui s'imposait réellement, à savoir l'uniformité des titres des pièces sur la base du nom des instruments, n'a pas été apportée. C'est ainsi que l'on retrouve dans la nouvelle édition des titres reflétant le nom d'un instrument, à côté d'autres titres se rapportant au mode de jeu (par exemple, "la ronde des écus») et d'autres encore qui indiquent l'occasion musicale (comme le "Zimmermannsstreich»). Quant aux traductions, elles manquent de cohérence ; ainsi le terme allemand Schellenbaum désignant le « chapeau chinois » figure$\mathrm{t}$-il seulement entre parenthèses après l'appellation française. Quand on revendique une terminologie scientifique, il faut s'y tenir avec rigueur.

7 Une autre carence de la réédition est la suppression du texte italien, alors que plusieurs exemples musicaux proviennent de la Suisse italophone. Une publication qui veut rendre compte de toutes les régions linguistiques de notre pays devrait être multilingue, d'autant plus que l'édition antérieure en donne l'exemple.

Dans l'introduction de la notice accompagnant le CD réalisé lors de la Journée de la musique populaire suisse, célébrée le 4 septembre 1994 dans le cadre du Festival international de la musique à Lucerne, Brigitte Bachmann-Geiser affirme au sujet de la diversité culturelle et linguistique de la Suisse: "En Suisse, les différentes cultures s'expriment dans les quatre langues nationales et dans de nombreux dialectes [...] Les régions se distinguent non seulement par leur structure géographique, leur langue [...] mais aussi par leur musique [...] Il est encore possible de nos jours de classifier la musique traditionnelle suisse en termes régionaux». L'objectif principal de la Journée de la musique traditionnelle suisse était de démontrer, avec le concours de musiciens et de chanteurs venant de toutes les régions de Suisse, que la musique traditionnelle suisse se perpétue en dépit d'une folklorisation et d'une commercialisation omniprésentes. Les enregistrements figurant sur le $\mathrm{CD}$ en question, réalisés live par un ingénieur du son du Studio de Bâle de la Radio suisse-alémanique, établissent ainsi un lien avec le CD publié par Claves. Tandis que ce dernier atteste la pluralité vivante des instruments de la musique traditionnelle suisse, le CD lucernois illustre les divers styles régionaux de cette musique.

Dans le second cas également, l'auteur était responsable du choix du programme et du commentaire. Au cours de ses longues recherches sur les instruments de musique, elle a fait la connaissance de nombreux interprètes de musique traditionnelle de toutes les régions de Suisse, dont elle a pu inviter un grand nombre à Lucerne. Elle a su assurer une programmation harmonieuse en engageant à la fois des musiciens - y compris des enfants - qui pratiquent une musique régionale traditionnelle et de jeunes artistes qui se 
consacrent à la réinterprétation de la musique et des chansons traditionnelles ; parmi ces derniers, mentionnons l'ensemble tessinois "Vent Negru », la comédienne et musicienne bernoise Christine Lauterburg qui pratique le yodel, le duo musette des accordéonistes jurassiennes Corinne et Fabienne Chapuis, et enfin Mytha, le «Contemporary Alphorn Orchestra».

Cette Journée de la musique traditionnelle fut un événement inoubliable car elle offrit l'occasion rare de faire l'expérience, en une seule journée et en un seul lieu, de toute la diversité vivante de la musique traditionnelle suisse. Selon le programme, une heure environ était réservée à chaque région. Brigitte Bachmann-Geiser introduisit les différents groupes de façon compétente et claire, tout en cédant la parole aux interprètes qui voulaient eux-mêmes commenter leur musique. Des extraits du programme furent présentés le jour même et le lendemain par la télévision et la radio suisses, et la manifestation trouva également un écho dans la presse écrite. Mais il aurait été dommage que cette image sonore unique de la musique traditionnelle suisse n'ait pu servir à d'autres fins.

Inspirée par le nombre de réactions positives, Bachmann-Geiser a œuvré pour que les enregistrements en direct de la Radio DRS et une sélection des meilleurs clichés réalisés par les photographes de presse fassent l'objet d'une publication. Elle a trouvé un éditeur de choix en la maison Zytglogge, connue de longue date pour ses activités dans le domaine de la promotion du patrimoine culturel suisse, qu'il prenne la forme de l'écrit, de l'image ou du son.

Les trente-sept chansons et danses de ce $\mathrm{CD}$ ont été réparties en fonction des styles régionaux. La première partie comprend des exemples typiques de Suisse centrale, comme le jeu d'instruments simples, souvent fabriqués par les interprètes-amateurs euxmêmes, et diverses formations caractéristiques (notamment un ensemble de " cithares à manche » de Kriens), auxquels s'ajoutent le jeu du Büchel et le yodel naturel du Muotatal (canton de Schwyz). Les autres régions sont représentées sur le même modèle, par leur musique vocale et instrumentale distinctive il s'agit de la région bernoise, de celle du Säntis, du pays de Glaris, des Grisons, du Tessin et de la Romandie. Dans la notice d'accompagnement, une description détaillée des caractéristiques musicales de chaque région fait figure d'introduction, suivie d'indications précises, pour chaque pièce, sur les interprètes, les instruments joués et, le cas échéant, le contenu de la chanson. Pour aider à visualiser l'étendue respective des différentes régions concernées, une carte géographique de la Suisse est reproduite en première page.

Tout ce matériel est complété de manière exemplaire par la documentation que l'auteur a réunie sur les instruments de musique traditionnels suisses. Nous disposons ainsi d'une image sonore authentique et exhaustive de la musique traditionnelle suisse dans toute sa vitalité et sa diversité régionale. Ces qualités sont soulignées par la prise de son en direct, qui nous livre les réactions et les applaudissements du public. C'est un CD excellent sur tous les plans mais on aurait souhaité une traduction française et italienne de la notice d'accompagnement, non seulement pour respecter la tradition suisse du multilinguisme, mais encore pour rendre hommage à la contribution importante des interprètes originaires de ces régions linguistiques. 